\title{
17 \\ THE ROLE OF COMPETENCIES AND INTERESTS IN DEVELOPING COMPLEX INFORMATION TECHNOLOGY ARTEFACTS: The Case of a Metering System
}

\author{
Diego Ponte \\ Alessandro Rossi \\ Marco Zamarian \\ Università degli Studi di Trento \\ Trento, Italy
}

\begin{abstract}
This paper contributes to the ongoing debate on the relationship between artefacts and organizational structuration by describing the dynamics surrounding the collaborative development of information technology artefacts. The research addresses a clear gap in the literature, as cooperation in artefact design has rarely been analyzed. To explore this issue, we analyze as a case study the various attempts, undertaken by a consortium of various economic actors, at developing an electronic metering system. The main results emerging from the field study are (1) the relevance of each actor's interests as the main rationale for explaining the technical features of the artefact, (2) the role of negotiation and consensus in determining the final shape of the artefact in term of its features, and (3) the bundling/unbundling of features within the physical object as the cooperative effort rises/falls.
\end{abstract}

Keywords Artefacts, interests, ambiguity, competencies

\section{INTRODUCTION}

In the last few decades information technology artefacts have been attracting the attention of organizational scholars. The reasons behind this attention are twofold. First, the role and importance of IT artefacts (thereafter simply called artefacts) in the current

Please use the following format when citing this chapter:

Ponte, D., Rossi, A., and Zamarian, M., 2008, in IFIP International Federation for Information Processing, Volume 287, Open IT-Based Innovation: Moving Towards Cooperative IT Transfer and Knowledge Diffusion, eds. León, G., Bernardos, A., Casar, J., Kautz, K., and DeGross, J. (Boston: Springer), pp. 291-308. 
economy is growing fast. Second, the introduction of artefacts within an organization often implies the modification of current patterns of action and routines. Such an introduction usually influences the layout of a firm and might be in contrast with the current way of doing things (Orlikowski 2000).

The increasing pace of technology evolution in recent years has made IT artefacts very complex objects: they embed multiple technologies and might pursue numerous complex tasks without human intervention. The reverse side of this complexity is that firms find it too much of a challenge to possess and manage all of the necessary knowledge that is needed to design and produce such artefacts. Thus, it is becoming more and more common for firms to rely on formal and informal agreements with heterogeneous partners to cooperate and to mutually complete the respective capabilities and competencies. As a consequence, conventional models, methods, and tools used to support artefact design and implementation are becoming obsolete.

From this latter point, we feel that current research is in need of a deeper understanding of the processes underlining artefact development within a network of actors (firms, research institutes, and the public sector). In fact, while it is well known that within an organization the development of new products and artefacts is a complex and tough task, this task grows in complexity when considering several nonhomogenous actors: the knowledge, language, competencies, and identities at stake might be very different and even inconsistent. Furthermore, as artefacts have been considered as reifications of the firm's competencies and knowledge, the development of an artefact by a network of firms poses the question of how different firms with different competencies will cooperate and negotiate the creation of a common framework to develop the artefact.

The paper describes and discusses the evolution of the design of a specific artefact: an electronic metering system. This artefact has been the topic of research within a consortium of firms operating in the green building/renewable energy business. The results of the analysis show that the conceptualization and evolution of the artefact was mainly driven by the interests of the actors at hand rather than by pure technical problems and concerns. First, the case study shows that the vision of the artefact's features and goals evolves as the actors working on it change over time. Second, this evolution is dependent only on the firms' interests in preserving their competencies and capabilities. A major corollary of this study is that while the technology allows the conceptualization of several possible configurations of the artefact's functionalities, the final configuration is chosen in a way that is consistent with the interests and competencies of the actors at hand. These findings make it possible to shed new light on the social processes that characterize the negotiation process around an artefact.

\section{IT ARTEFACTS: BETWEEN DESIGN AND USE}

IT artefacts have been defined as objects that embed sets of rules for goal-oriented action (Norman 1991, 1993). This definition allows us to identify some specific characteristics of artefacts. First, they are usually considered goal-oriented tools: they are designed to solve problems and help achieve some particular tasks and actions (Hutchins 1995). As such, artefacts often help lower the cognitive complexity of a particular activity: thus they are considered to be crystallized solutions for recurrent problems. 
Second, as they embed already developed routines and solutions to current problems, artefacts tend to structure and shape organizational behavior. Although artefacts might be customized by users, a kind of behavior which has been called instrumentation (Rabardel 2003), their purpose in curbing the complexity of a particular task implies that such customization can be fulfilled only partially. In particular, users can rarely change the artefact's core.

Third, as artefacts embed solutions to some particular problems, they are considered objects that reify the knowledge and the competencies of the individuals designing and using them (Hutchins 1995). Furthermore, artefacts become part of the tools that are significant from an organizational perspective because they incorporate rules for negotiating between differing, and at times conflicting, points of view (Masino and Zamarian 2003).

In the current literature on artefacts, design and use are usually treated as different phases with different actors (experts versus users), inputs (the problem versus the artefact), outputs (the artefact versus the accomplishment of a particular task), and purposes.

Historically, analysis of artefacts began by looking at their diffusion within an environment; the design phase was barely taken into consideration (Arthur 1989; David 1986). In the last few decades, this situation has rapidly changed (Dosi 1992). Currently, scholars tend not to separate the evolution of an artefact into rigid phases: design and use are not detached (Dosi 2000; Gherardi and Nicolini 1999). This change of focus in the analysis is linked to the growing recognition that designing an artefact should be considered as a shared activity among a set of interacting actors (designer and users) (Béguin 2003; Béguin and Rabardel 2000; Bødker 1996).

Recently, this latter consideration has been put forward as a step toward recognizing that the design phase might be a phase where different heterogeneous actors and organizations interact. This might happen in situations where actors must cope with new and complex technologies, unclear problems, and innovative solutions (Albinsson et al. 2007).

Focusing on how different organizations try to collaborate to develop an artefact opens up the opportunity for new research. In fact, while it is well known that within an organization the development of new products and artefacts is a complex and tough task (Bechky 2003; Carlile 2002), this task grows in complexity when considering the interaction of several heterogeneous actors. In this latter case it is well known that knowledge, language, competencies, values and identities of the interacting actors might be idiosyncratic (Gherardi and Nicolini 1999; Woolgar 1991). Unfortunately current research has barely addressed such developments (Beck 2002). Scholars have focused on analyzing either the interaction between design and use of artefacts within individual organizations or the processes of interaction between several kinds of actors within each of the two processes (Chesbrough 2003; Perry and Sanderson 1998 ). Other studies have suggested the adoption of best practices, which unfortunately are not always carefully operationalized (Docherty 2006). Furthermore, other studies focus on sector-level analyses, which do not offer a rich understanding of the micro-factors influencing design and implementation (Laursen and Salter 2006).

We try to address some of these concerns by analyzing the dynamics surrounding the attempts at creating an innovative, complex artefact: an electronic metering system, developed within a private/public consortium operating in the green building/renewable energy business. This case study is relevant for at least three reasons. First, the artefact was a complex object made of heterogeneous parts (software and hardware) that implied 
the use of different technologies (wired communication, wireless communication) owned by different actors, representing various companies and research institutions. Second, the team of designers was made up of several heterogeneous actors (firms, research institutes, and the public sector). This team evolved over time, so consequently the goal of the artefact evolved over time. Third, the artefact did not have a commonly agreed upon and well-understood goal. In fact, more than just a set of core services (management of power consumption flows), the metering system was intended to cover a set of disparate peripheral services such as the management of gas and water consumption as well as domotics and communication services.

\section{THE CASE STUDY}

The context of our case study is a consortium of firms operating in the green building/renewable energy industries. The consortium, located in a highly developed region of an EU country, was born out of the voluntary initiative of private corporations and various other stakeholders with the goal of facilitating and fostering the innovation and collaboration activities of local firms working in various environmentally sustainable industries.

We focused on the network workgroup initiative, which is a workgroup aimed at designing and developing innovations in the business of local communication infrastructure. The main task of the workgroup, at the time, was that of designing an artefact that took care of the management of incoming/outgoing data fluxes of a building (gas, power, water, etc.).

In order to clarify the relationship between the different actors, in the next paragraph we characterize the institutional framework within which these companies operate; then we outline the main events accounting for the evolution of the artefact.

The Consortium. In March 2005, Local Government, in agreement with various economic institutions (such as the local Development Agency and the Chamber of Commerce) and various local research centers and universities, signed an agreement aimed at developing a regional "Pole of Excellence" in the green building/renewable energy business. As a result, Local Government supported the creation of a public-private Consortium, comprising over 300 private corporations as well as the most important public institutions. The official aim of the Consortium is that of supporting collaboration and innovation between its various stakeholders both at the local and global level. The Consortium began its activities in 2006. In particular, the Consortium focused on topics such as the construction of green buildings (energy efficient buildings), on high efficiency heating systems, on intelligent systems for the management of water, power, and communications flows, and on systems for the production of renewable power.

In each of these areas, the Consortium organized a series of initiatives to facilitate collaboration, the transfer of knowledge, and innovation among its stakeholders. Four of them were particularly important:

- $\quad$ Set up and management of technical workgroups. These workgroups aim at facing and solving specific problems and topics that are related to the green building/ renewable energy sectors. It is to be noted that each workgroup's activities are 
managed by a facilitator, a Consortium-selected manager who takes care of all of the practical and strategic issues of the workgroup.

- Training initiatives, aimed at raising competencies and professional roles around specific technical standards or new regulations.

- Management of public relations at the aggregate level (brand management, participation in international fairs and seminars, etc.).

- Special services dedicated to the support of innovation and the creation of new services and products.

The network workgroup belongs to the first of these activities. The next paragraph describes the evolution of the network workgroup.

The Network Workgroup. As mentioned earlier, one of the tasks of the Consortium was that of establishing several technical workgroups. The network workgroup was mentioned in the official documents of the Consortium soon after its start-up in 2005. The goal of the workgroup revolved around the idea of supplying the local area with a fully coordinated communication network that would allow "objects" (buildings, infrastructures, etc.) to "communicate with each other" with the aim of improving efficiency in the consumption of resources (power, water, gas, etc.) and of streamlining various other services, such as domotics, social assistance, etc. Out of the 300 individual members of the Consortium, 29 have taken part in the network workgroup.

Figure 1 sketches a graphical representation of the main actors involved in the debate on the innovative artefact and distinguishes between actors directly participating in the workgroup activities and those influencing the workgroup activities even if not directly involved in its activities. ${ }^{1}$ Circles identify single agents while octagons identify members representing several actors (e.g., consortiums). The size of each circle and octagon identifies the bargaining power of each actor (either directly involved in the workgroup or not). The assessment of bargaining power is described in the methodology section. In short, the most powerful actors among those participating in the workgroup were the economic branches of the local government (LocalEnergy and LocalNet). LocalEnergy manages the local power network while LocalNet manages the public communication infrastructure.

Among the private participants, the most influential were the local consortiums of firms working in heating systems maintenance (HSMA Group), in the telecommunication sector (Com Group), and in the production and distribution of power (Power Group). Other less important actors involved in the workgroup were Houseit (a multinational in the home appliances business), X-Info (a local mid-size informatics firm) and a set of spin-off companies: TeleExp (a research center in telecommunication technologies), MicroElec (a microelectronic firm), and CareSolution (a remote health care service firm).

The figure also shows the external actors, who, although not directly involved in the workgroup, influenced and shaped the evolution of its activities. The external actors influencing the workgroup were NationalEnergy (the largest energy supplier of the country), Infomatic (a worldwide IT solutions provider), Local Government, and two political branches of Local Government (Social Affairs and Innovation Affairs offices).

${ }^{1}$ For reasons of privacy, the real names of the firms involved in the case study have been changed. 


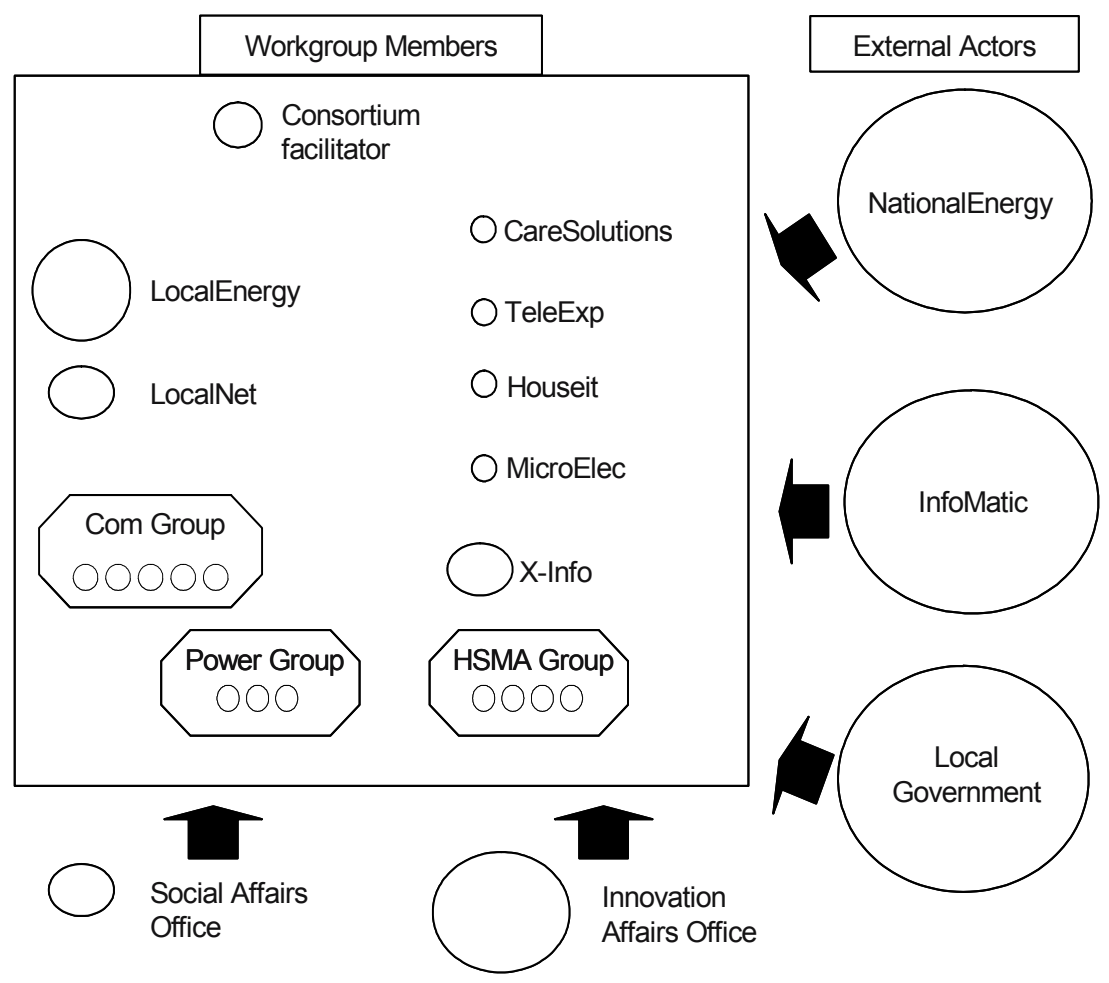

NOTES: The circles identify single actors. The size of the circle identifies the power of each actor. The octagons identify multiple actors (e.g., consortia of firms that work in the same sector). The number of circles within each octagon approximates the number of members of each consortium.

Power Group: firms working within the energy sector (supply of energy, maintenance).

Com Group: firms working within the telecommunication sector.

HSMA Group: firms working within the heating systems maintenance sector.

Figure 1. Participants of the Network Workgroup and External Actors Influencing the Innovation Debate

\subsection{Methodology}

The complexity of the case required the adoption of a qualitative methodology (Denzin and Lincoln 1994). In particular, the data collection was divided into two main phases. The first phase aimed at understanding the economic-institutional context in which the case study took place. This phase, performed during the first part of the 2007, was grounded on second-hand materials such as newspaper articles, official and technical papers, and institutional materials.

The second part of the data collection focused on the actual activities performed by the workgroup. The goal of this part was that of gathering data about the evolution of the 
artefact and the elements influencing such evolution. This part of the research was based on:

- A series of interviews with key actors of both the workgroup and the Consortium. We used structured interviews, as we wanted to focus on a specific experience (Silverman 2001): we wanted to confine the discussion to several aspects surrounding the artefact, the workgroup dynamics, and the actors involved.

- Participant observation of the activities of the workgroup (Atkinson, and Hammersley 1994; Merton et al. 1990). One of the researchers actively took part in the meetings of the workgroup. In particular, the researcher took part in several meetings that were concerned with the workgroup. This participation made it possible to track the dynamics among the different (and changing) actors.

- Document analysis. We acquired several internal documents such as agendas and minutes of meetings, as well as institutional material distributed by the Consortium.

Table 1 summarizes interviews and official meetings attended. The output of this phase was a report of the artefact's evolution and the actors involved over the period from a historical perspective.

The analysis of the data was split into two main phases. The first phase aimed at assessing the bargaining power of each actor. We also sought to establish the reasons (the interests) that motivate each actor to participate in the workgroup. The assessment of bargaining power was determined via a two-step procedure. First, we analyzed the business, market, and regulatory characteristics of each involved actor. For instance, we assumed NationalEnergy to have a high bargaining power as it controls over 80 percent of the national power distribution market. We also considered Local Government and LocalEnergy to have a high bargaining power (although less than that of NationalEnergy) as they govern and control the local power distribution market. We then considered the other actors as having less bargaining power, as they do not hold dominant positions. During the second step we adjusted this first assessment by means of the post hoc interviews and by taking part in the workgroup activities.

\section{Table 1. Review of the Material Used During the Analysis}

\begin{tabular}{|l|l|}
\hline \multicolumn{2}{|c|}{ Meetings Attended } \\
\hline $29 / 05 / 07$ & Network Workgroup meeting \\
\hline $04 / 06 / 07$ & Consortium plenary meeting \\
\hline $05 / 10 / 07$ & $\begin{array}{l}\text { IDDC (Innovation in Development and Design of Constructions) } \\
\text { Committee preliminary meeting }\end{array}$ \\
\hline $08 / 10 / 07$ & Network Workgroup meeting \\
\hline $08 / 11 / 07$ & Network Workgroup meeting \\
\hline \multicolumn{2}{|l}{ Interviews } \\
\hline $10 / 05 / 07$ & Former facilitator of the workgroup \\
\hline $11 / 07 / 07$ & Current president of the Consortium \\
\hline $01 / 10 / 07$ & Current facilitator of the workgroup \\
\hline $24 / 05 / 07$ & CEO of the Consortium \\
\hline
\end{tabular}


The second phase of the data analysis aimed at triangulating all available data in order to establish whether the negotiation dynamics and the evolution of the artefact were driven by the interests of the actors involved. The inputs of this phase were the economic-institutional report, the historical report, and the bargaining power report as previously introduced. We were thus able to see whether the evolution of the artefact's interpretation was subject both to the indirect bargaining power indicator and to the more direct dynamics of the workgroup meetings. In the next section we briefly review the main events of the case study and discuss the findings of our analysis.

\subsection{Time Line of the Main Events}

This section describes the history and the evolution of the dynamics surrounding the conceptualization of the artefact under discussion during our analysis. The time line starts with a description of a prior, similar project carried out by NationalEnergy. It goes on to detail the three different stages of the evolution of the metering system (Figure 2).

\subsubsection{Before 2006: The Origins}

The artefact under analysis has roots in an innovation project started by National Energy, a leading power supply company. In the final years the past century, National

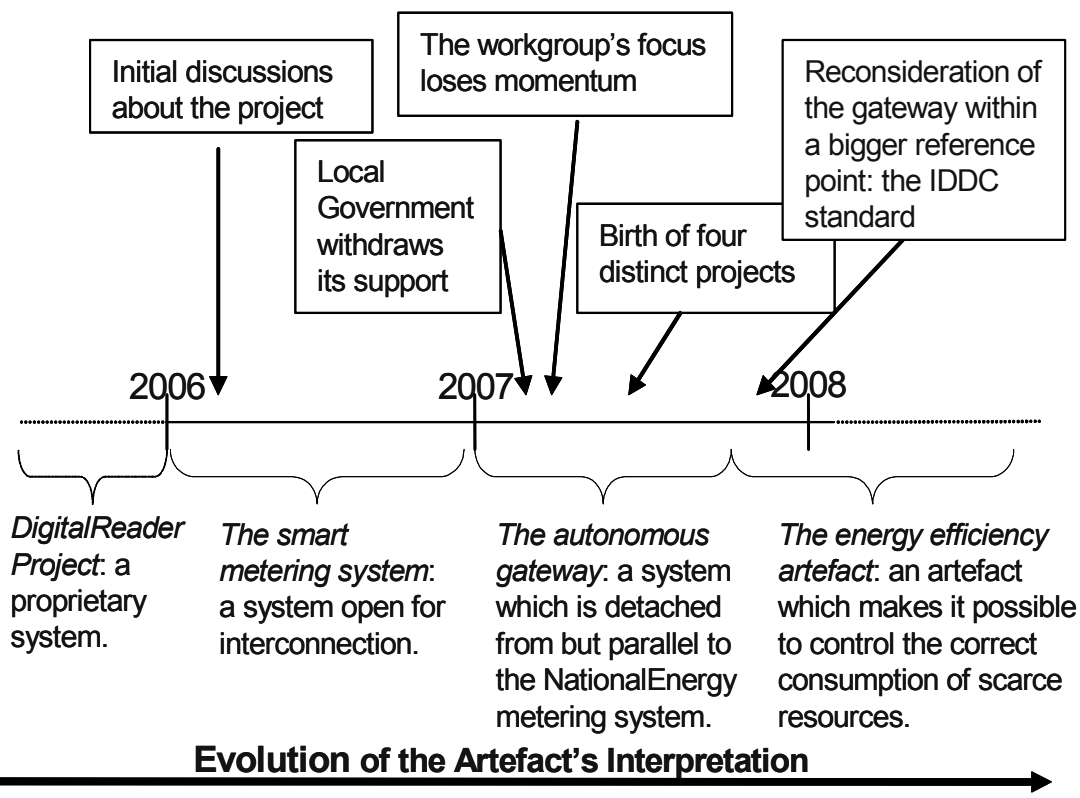

Figure 2. Review of the Case Study Time Line and Description of the Interpretive Frameworks Used to Describe the Artefact 


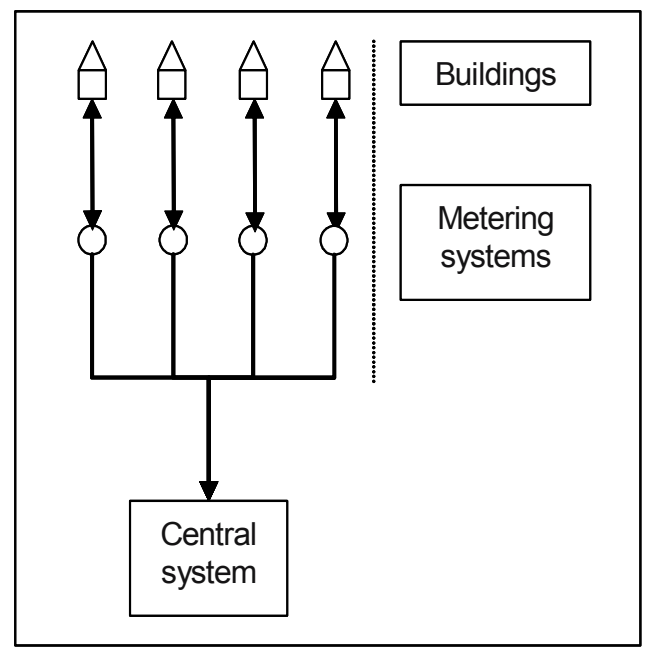

Figure 3. Outline of the DigitalReader Architecture

Energy started a project aimed at introducing the first worldwide digital metering system (hereafter called DigitalReader) as a replacement for the old electro-mechanical metering system. The research activities concerning the DigitalReader, being quite challenging, took almost 10 years from the first conceptualization to its installation. The Digital Reader (see Figure 3) was designed as a metering system able to:

- Manage data coming from both the central system and from the buildings: the system not only collected data about power usage, but also about water consumption and communication exchanges from other sources.

- Make automatic decisions and actions aimed at improving the efficiency of resources consumption. This task should have been performed - and this is the main innovation-without the direct intervention of the central system.

Due to these features the metering system was labeled "smart" (thereafter referred to as smart DigitalReader).

NationalEnergy interacted with two main high technology partners for both the design and industrialization phases of the artefact. The first partner, Chiplon, was an innovative firm engaged in the production of smart chips. Chiplon supplied National Energy with a high-tech chip that is the reason behind the improved capabilities of the smart metering system. The second partner was a multinational firm based in Europe that was responsible for the production of the metering system. The rest of the project team consisted of internal personnel.

Starting in 2001, NationalEnergy began to install the metering system. While the original device was recognized as a worldwide innovation, NationalEnergy decided to install a dumbed-down version of that system that was lacking the innovative capabilities of the smart one (automatic decisions, no need of a centralized system). The dumbed- 
down DigitalReader was thus presented as a digital metering system lacking the feature of interacting with the buildings. NationalEnergy also put a stop to the collaboration with Chiplon claiming that Chiplon was working on a concurrent project with other companies. Today the DigitalReader project is over, as far as its development is concerned. The dumbed-down DigitalReader has been installed in most of the country: in fact, more than 80 percent of the country's territory is covered by this system.

As previously mentioned, the evolution of the DigitalReader project is important as it sheds light on what happened within the workgroup. The electronic metering system was in fact not installed in the local area as the network - managed by LocalEnergy, a firm owned by Local Government - still relies on first generation, electro-mechanical devices.

\subsubsection{6-Spring 2007: The New Metering System}

Starting in 2006, the workgroup began to actively work at the idea of an electronic metering system. Several actors (public institutions, firms, research institutes) participated in the activities of the workgroup. In 2006, during a conference organized by the Consortium, the facilitator of the workgroup launched the idea of introducing in the local area a metering system similar to the one previously discarded by NationalEnergy (the smart DigitalReader). The aim was to develop a real information gateway able to communicate both with the devices within the building and with a central system that belonged to the multi-utility company. In contrast with the solution implemented by NationalEnergy (the dumbed-down DigitalReader), which used a proprietary protocol, this gateway was intended to operate under an open communication protocol. Thus, it was open to interaction with other systems.

At this stage, several actors supported the system. This is due to the fact that the electronic metering system would have allowed several peripheral services. The workgroup identified four different types of features, each supported by different sets of actors:

- Remote maintenance of home appliances. This feature was sponsored by Houseit, a home appliance multinational.

- Remote management services. This project, led by the heating systems maintenance association (thereafter HSMA group), includes several subgoals:

- The first set of services was intended to manage the remote-reading of water and gas consumption

- The second set of services was intended to support security services.

- The third set of services aimed at developing a web-based interface of the aforementioned services.

- Remote health care. This subproject, led by the spin-off company CareSolutions, was intended to function as a remote channel to provide services to the elderly and individuals who were not self-sufficient. This project was also supported by the local government social affairs committee.

- Domotics. This subproject was promoted by MicroElec, a microelectronics firm, and was intended to offer in-house services such as the remote management of the internal temperature and humidity, as well as fire prevention services. 
In 2007, the initiative was presented to a local software firm, which was partly owned by InfoMatic. InfoMatic was not a participant either in the Consortium or in the workgroup, but became aware of the local project. It is worth noting that there is a business relationship between InfoMatic and NationalEnergy. InfoMatic was in fact supplying NationalEnergy with the software of the dumbed-down DigitalReader system. After this news, InfoMatic started lobbying in the local area with the aim of derailing the project. InfoMatic also informed NationalEnergy about the project.

\subsubsection{Spring 2007: The Short Life of the Gateway}

Thanks to the lobbying activities of InfoMatic, the project to introduce a smart metering system experienced a sudden halt. Once InfoMatic (and thus NationalEnergy) showed its opposition to the project, the local power supply company, LocalEnergy, withdrew its support. Indeed, a clarification meeting with the local government (owner of LocalEnergy), made it clear that they were opposed to the project as well, claiming that the rest of the country was using the dumbed-down DigitalReader artefact and that they were looking for a robust and reliable system rather than an innovative one.

Once the support of the local government and LocalEnergy was withdrawn from the project, the workgroup experienced a chaotic period. At that time, the goal of the workgroup became unclear. In fact, without the support of these actors the system could not be introduced in the local area. At first, some of the participants sought to go further with the project, calling it a gateway. The CEO of LocalNet and the manager of TeleExp were thinking about an information gateway, autonomous and separate from the NationalEnergy metering system. This parallel system would use an open communication protocol. The promoters pushed this idea on the grounds that the local government had put a lot of effort into developing a communication infrastructure throughout the whole territory (indeed LocalNet was in charge of implementing and managing this infrastructure). Their idea was that, if a local multi-utility was willing to support the project, perhaps the project could gather the necessary support from other actors. Unfortunately, this idea did not achieve any momentum. In fact, during the spring of 2007, the activities of the workgroup once again came close to a halt. Nonetheless, the relative ambiguity of the workgroup goals, and the confusion during spring 2007, lead to the start of four independent subprojects during the summer.

\subsubsection{The Birth of Four Subprojects}

The chaotic phase of the workgroup saw the birth of four different research proposals. As the group had lost some of its members, the facilitator of the workgroup and the managers of the Consortium were noticeably surprised at the growing number of proposals submitted concerning the artefact. These projects were promoted by different actors, none of whom was aware of the other projects.

The collaboration with LabTech and MultiPower. LocalNet and TeleExp, having failed to attract the necessary support from the workgroup, constituted the first autonomous subproject, aimed at producing a working metering device. This group was interested more in a simple object that could work promptly than in a comprehensive 
communication and data management project. The goal was that of discovering the real costs and practical functionalities of such an artefact.

This project was supported by a micro-electronics company, LabTech, a producer of components for the communication sector. The participants also wanted to assess the sustainability of such a project by means of field tests. The opportunity for performing a test was supplied by a multi-utility, MultiPower. This company supplies water and gas power in a nearby extensive metropolitan area. MultiPower was involved as they were already working on a very similar project.

The three minor projects. In parallel to the aforementioned project, three separate sets of firms presented three proposals to the managers of the Consortium. The peculiarity of these proposals is that they were autonomously prepared by each coalition, bypassing the role of the Consortium managers. These proposals were focused on different types of business opportunities and show a clear lineage with the projects presented in the first stage of the workgroup. The three projects were Remote Management Services (sponsored by the HSMA group), Remote HealthCare Services (led by CareSolutions), and Domotics (sponsored by the microelectronic firm MicroElec).

The outcome of these projects is at present unclear, since they are still in progress. In effect, they seem to suffer from serious lack of leadership, since they are managed neither by the facilitator of the workgroup nor by other managers of the Consortium. Nonetheless, it seems that the scope of these latter projects is exploratory in nature rather than pertaining to a tangible new business initiative.

\subsubsection{Recent Developments of the Workgroup: The IDDC Standard}

Since October 2007, the workgroup has shown renewed commitment to a common goal. This impulse is the result of external input. In this period, the Consortium presented its new strategic agenda for the next 10 years. This document sets out the agenda for fostering the development of green and sustainable businesses within the local area. Moreover, it sets the new guidelines to achieve such a goal. As an example, the Consortium decided to adopt, in agreement with the local government, the IDDC (Innovation in Development and Design of Constructions) ${ }^{2}$ green-building standard as a reference point for the construction of new buildings in the local area. Here the Consortium decided to apply for the possibility to become the national reference-point of the IDDC certification.

In this context, the metering system has been proposed anew as an essential tool to efficiently manage the flux of data coming in and out of the buildings. While this is the purpose, it is not possible to describe the evolution of the situation as it is still evolving.

\section{ANALYSIS}

In this section, we discuss the behavior and choices of the different actors in an attempt to describe the rationale for their decisions about the artefact.

${ }^{2}$ The IDDC standard is an international protocol that includes a set of principles for the design and production of green buildings. 


\subsection{Until 2006: The DigitalReader Project}

The behavior of NationalEnergy, although not the focus of this paper, deserves some attention. In fact, while its smart DigitalReader project was considered a worldwide innovation, NationalEnergy decided not to install it in favor of a clearly inferior device (the dumbed-down version of the original project). NationalEnergy also stopped the collaboration with Chiplon claiming that Chiplon was working on a competing device with other partners. From our point of view, the reason behind this decision might be found in the fact that Chiplon asked NationalEnergy to adopt an open communication protocol for its metering system while NationalEnergy wanted to use (and eventually adopted) a proprietary protocol. These inconsistent views about an important aspect of the metering system might be the real cause of the end of the cooperation. On the one hand, Chiplon wanted an open protocol so to have an interoperable device, while NationalEnergy wanted a proprietary device so as to avoid any possible interaction with other systems. And the position of NationalEnergy was irremovable. As a matter of fact, this behavior resulted in the deterioration of the relationship with Chiplon and with the decision to install the dumbed-down version of the DigitalReader.

\subsection{6-2007: The Smart Metering System}

As already mentioned, the project of a smart metering system came to a stop due to the lobbying activities of InfoMatic. We need to underline here that the resistance was not technical in nature. The local area was still using the old electro-mechanical metering device while the installation of the new system had been planned already (about 400,000 pieces). This was a compulsory task as a recent directive of the National Power Regulation Authority urged the power supply firms to introduce an electronic metering system. For obvious reasons, the natural candidate to be installed in the local area was the dumbed-down DigitalReader (which had already gained a market share of over 80 percent).

The features of the metering system that was developed locally, as a second candidate, would have been clearly in contrast with the dumbed-down DigitalReader (which, again, had been developed by NationalEnergy in cooperation with InfoMatic, who provided the software): these characteristics might be summarized by the openness of the system protocol. The NationalEnergy/InfoMatic artefact was based on a closed communication protocol while the project of the workgroup would have adopted an open protocol, as it was in the best interest of the independent third party service providers.

The strong lobbying activities of InfoMatic convinced the local government, and thus the local power supplier (LocalEnergy) to give up their support of the project. Local Government justified its behavior by arguing that the workgroup proposal was too innovative and hazardous from an economic point of view. Indeed, Local Government and the local power supplier (LocalEnergy) were seeking a robust and longstanding artefact while the other participants of the workgroup liked the possibility of working on the innovative aspects of the artefact. 


\subsection{7: The Gateway and the Birth of Several Subprojects}

This phase seems to be the most intriguing as, once the support of the main local actor-Local Government—vanished, the workgroup faced serious problems. This is due to several reasons:

- There was no main actor with enough strength to dominate the negotiating workgroup.

- The withdrawal of LocalEnergy from the project resulted in the disappearance of an important test market for the device.

- The interests of the remaining actors were pulling in different directions.

- As a consequence, there was no clear understanding of what to do.

The combination of these factors lead the different coalitions belonging to the workgroup to work toward devices that incorporated only those feature that enabled their personal business opportunities. Obviously the easier way to achieve this goal was that of an unbundling of the characteristics of the original artefact. The problem for each of the coalitions, then, became attracting the interest and commitment of a utility network manager (the prospective adopter for the artefact, which in the previous phase was represented by LocalEnergy). So, while the general framework of the system was clearproducing an artefact able to gather, manage and send information in and out of a building - the four projects were modeled around specific core business and partnerships.

\subsection{End of 2007: The IDDC Standard}

The more recent activity of the workgroup (fall 2007) shows that the managers of the Consortium were trying to understand whether participants were keen on continuing the collaboration. Past history showed that, without a common goal and a leading actor, the workgroup dispersed and became purposeless. The IDDC standard provided the facilitator of the workgroup with a new opportunity to commit to a clear agenda. This standard was chosen as the new green-building policy of Local Government.

From the political point of view, the Consortium was attracted by the possibility to become the national reference point for the standard. The network workgroup is actively working on the possibility of introducing an electronic metering system as a necessary control system of buildings' consumption fluxes within the standardization protocol.

\section{DISCUSSION}

The environment in which the analysis was performed is the network workgroup, a place where actors of different backgrounds sought to collaborate toward a common goal: developing a smart electronic metering system.

The analysis showed that the workgroup underwent several contrasting events. The interpretation, characteristics, and features of the artefact evolved with the change of the 
involved and noninvolved actors. The main actors in the first period-NationalEnergy, InfoMatic, and Local Government - were the most influential actors in the workgroup. The withdrawal of their support from the initial project signed its immediate death warrant. The remaining actors did not have enough (economic and political) strength to put forward the project from a global point of view.

At the time that the leading actors left the group, the vision of what the artefact should do changed. Originally the metering system was conceived as a worldwide innovation similar to the one developed by NationalEnergy. Unfortunately the lobbying activities of InfoMatic, and thus NationalEnergy, who were not members of the workgroup, caused Local Government (and its operative branches) to block the project. After this halt, different alliances of firms submitted several independent subprojects. Although there is little information about these projects, it seems that only one of them saw the in-field experimental phase. This experimentation was only interested in analyzing the "pure" technical aspects of the artefact while the introduction into the market was a secondary aspect.

Finally, the workgroup gained renewed momentum thanks to the IDDC standard, which gave a new framework to make sense of and gather consensus on the artefact. Unfortunately at this stage it is not possible to further the discussion as these latter events are still under analysis. To summarize, it is possible to outline several important aspects that the case study does show.

Political rather than technical aspects lead the design phase. The findings of this study allow us to state that the dynamics surrounding the conceptualization of the metering system (and its following evolution) were driven by political aspects rather than by technical concerns. In fact, it seems that in each phase the technical aspects of the artefact were selected $a d$ hoc on the basis of the interests (the political aspect) of the actors at hand. This evidence is further highlighted by the birth of the four different subprojects. Each of these projects was driven by the interest of the coalition/firm supporting it. The case study further shows that the plan of the workgroup was shaped by the intervention of external firms (i.e., firms that were not directly involved in the project). The lobbying activities of InfoMatic clearly show that the network workgroup was threatening its own interests.

Goal ambiguity of an artefact. The evidence also shows that the technological opportunity to attach several features to an artefact allows the involved actors to think of several plausible artefacts with different purposes. The artefact has been intended, alternatively, as (1) a traditional metering system, (2) an independent getaway for heterogeneous information flows, (3) a device (no matter whether smart or dumb) capable of supporting services provided by independent sources, and eventually (4) a device to optimize resource consumption. Each of these different interpretations of the artefact was supported by a different mix of actors. This latter consideration allows us also to state that the economic viability of a project is evaluated by the different actors on the basis of its ability to foster their specific interests and competencies. The case of the HSMA group gives us plenty of insight. Their interest in the project was based on its ability to remotely managing heating systems, thus dramatically curbing the maintenance costs.

Loss of leadership leads to unbundling. When the local government withdrew its support from the project, the network workgroup faced a chaotic period that resulted in 
several unrelated projects. The evidence shows that a leading actor, with its ideas and, moreover, with its bargaining power, might have an important aggregating function. In fact, until the leading actor (the local government) was involved in the project, the other less strategic and less powerful actors followed the main idea (the smart metering system) while trying to add to the artefact the "appropriate" features (i.e., the features in which they were interested). When the leading actor withdrew its support, the artefact was unbundled in several specialized projects, as none of the remaining actors possessed the necessary strength to maintain the cohesion of the group.

Artefacts and standardization. The case study shows that the conception of different devices with different purposes (the goal ambiguity), might become dangerous when particular conditions are met. In this situation, it is clear that the metering system was subject to the usual standardization dynamics: being basically a communication device, the artefact is subject to network effects and thus it can become the center of standardization wars (Cusumano et al. 1991; Dosi 2000; Stango 2004).

\section{CONCLUSIONS}

The analysis presented in this paper describes the dynamics surrounding the development of an artefact. The particularity of the case study is that the artefact has been developed within a network of heterogeneous actors. This is perhaps one of the main relevant aspects of this work as the mainstream literature barely analyzes such a collective way of designing artefacts. The findings of this analysis allowed the distillation of some important contributions to the current literature. We showed that the actors involved in the design of the artefact are more concerned with the political aspects of the artefact rather than with the technical problems to solve. Furthermore, we showed that without commitment or a common goal, the development of the artefact is a short-lived process as the goal-ambiguity, while it might be a useful tool to generate ideas at the earlier stages, subsequently causes confusion and impedes action.

This study suffers from some limitations. First, it is partial, as the dynamics surrounding the artefact are still in progress: the firms, the public sector, and the research institutes are still working on the artefact. As such, the process under study has not reached completion. It is, therefore, difficult to make clear cut comments about the results of the behavior of the different actors. Second, we aim at replicating this research, as replication is a required step to make in order to improve the quality and consistency of the findings (Yin 1994). One of the possible extensions regards the involvement of one of the other Consortium workgroups, which exist alongside the one analyzed in this paper.

\section{Acknowledgments}

The authors wish to thank Roberta Cuel, the Consortium CEO, and two anonymous referees for helpful comments on a previous draft. Financial support from MIUR (under the PRIN05 program) is gratefully acknowledged. The usual disclaimer applies. 


\section{References}

Albinsson, L., Lind, M., and Forsgren, O. 2007. "Co-Design: An Approach to Border Crossing, Network Innovation," in Expanding the Knowledge Economy: Issues, Applications, Case Studies, P. Cunningham and M. Cunningham (eds.), Amsterdam: IOS Press, pp. 977-983.

Arthur, W. B. 1989. "Competing Technologies, Increasing Returns, and Lock-In by Historical Events," The Economic Journal (99), pp. 116-131.

Atkinson, P., and Hammersley, M. 1994. "Ethnography and Participant Observation," in Handbook of Qualitative Research, N. Denzin and Y. Lincoln, (eds.), Thousand Oaks, CA: Sage Publications, pp. 249-261.

Beck, E. 2002. "P for Political-Participation is Not Enough," Scandinavian Journal of Information Systems (14), pp. 77-92.

Bechky, B. A. 2003. "Sharing Meaning Across Occupational Communities: The Transformation of Understanding on a Production Floor," Organization Science (14), pp. 312-330.

Béguin, P. 2003. "Design as a Mutual Learning Process Between Users and Designers," Interacting with Computers (15:5), pp. 709-730.

Béguin, P., and Rabardel, P. 2000. "Designing for Instrument-Mediated Activity," Scandinavian Journal of Information Systems (12), pp. 173-191.

Bødker, S. 1996. "Creating Conditions for Participation: Conflicts and Resources in Systems Design," Human Computer Interaction (11:3), pp. 215-236.

Carlile, P. R. 2002. "A Pragmatic View of Knowledge and Boundaries: Boundary Objects in New Product Development," Organization Science (13), pp. 442-455.

Chesbrough, H. 2003. Open Innovation: The New Imperative for Creating and Profiting from Technology, Boston: Harvard Business School Press.

Cusumano, M. A., Mylonadis, Y., and Rosenbloom, R. S. 1991. "Strategic Maneuvering and Mass-Market Dynamics: The Triumph of VHS over Beta," Business History Review (66), pp. 51-94.

David, P. A. 1986. "Understanding the Economics of QWERTY: The Necessity of History," in Economic History and the Modern Economist, W. N. Parker (ed.), Oxford, UK: Blackwell, pp. 332-337.

Denzin, N. K., and Lincoln, Y. S. 1994. Handbook of Qualitative Research, Thousand Oaks, CA: Sage Publications.

Docherty, M. 2006. "Primer on 'Open Innovation': Principles and Practice. The Next 'Big Thing' in Innovation,” PDMA Visions (30:2), pp. 13-17 (available at http://www.venture2. net/clientuploads/Visions_April06_Docherty.pdf).

Dosi, G. 1992. "Research on Innovation Diffusion: An Assessment," in Innovation Diffusion and Social Behaviors, A. Grubler and N. Nakicenovic (eds.), Heidelberg: Springer Verlag.

Dosi, G. 2000. Innovation, Organization and Economic Dynamics. Selected Essays, Cheltenham, UK: Edward Elgar.

Gherardi, S., and Nicolini, D. 1999. "La circolazione delle innovazioni come processo di traslazione," Studi Organizzativi (2), pp. 195-218.

Hutchins, E. 1995. Cognition in the Wild, Cambridge, MA: The MIT Press.

Laursen, K., and Salter, A. 2006. "Open for Innovation: The Role of Openness in Explaining Innovation Performance Among U.K. Manufacturing Firms," Strategic Management Journal (27), pp. 131-150.

Masino, G., and Zamarian, M. 2003. "Information Technology Artefacts as Structuring Devices in Organizations: Design, Appropriation and Use Issues," Interacting with Computers (15:5), pp. 693-707.

Merton, R. K., Fiske, M., and Kendall, P. L. 1990. The Focused Interview: A Manual of Problems and Procedures ( $2^{\text {nd }}$ ed.), New York: Free Press. 
Norman, D. A. 1991. "Cognitive Artefacts," in Designing Interaction: Psychology at the Human-Computer Interface, J. M. Carroll (ed.), Cambridge, UK: Cambridge University Press, pp. 17-38.

Norman, D. A. 1993. Things that Make us Smart, Reading, MA: Addison Wesley.

Orlikowski, W. J. 2000. "Using Technology and Constituting Structures: A Practice Lens for Studying Technology in Organizations," Organization Science (11:4), pp. 404-428.

Perry, M., and Sanderson, D. 1998. "Coordinating Joint Design Work: The Role of Communication and Artefacts," Design Studies (19), pp. 273-328.

Rabardel, P. 2003. "Editorial: From Artefact to Instrument," Interacting with Computers (15:5), pp. 641-645.

Silverman, D. 2001. Interpreting Qualitative Data: Methods for Analysing Talk, Text and Interaction, Thousand Oaks, CA: Sage Publications.

Stango, V. 2004. “The Economics of Standards Wars," Review of Network Economics (3), pp. $1-19$.

Woolgar, S. 1991. "Configuring the User, The Case of Usability Trials," in A Sociology of Monsters. Essays on Power Technology and Domination, J. Law (ed.), London: Routledge, pp. 58-100.

Yin, R. K. 1994. Case Study Research: Design and Methods (2 ${ }^{\text {nd }}$ ed.), Newbury Park, CA: Sage Publications.

\section{About the Authors}

Diego Ponte (Ph.D., Cognitive Science and Education, University of Trento) is a research assistant at the Faculty of Economics, University of Trento. In his dissertation he analyzed the socio-economic aspects that influence and shape organizational renewal. His research interests also include organizational decision making, organizational conflict, knowledge management, information systems, innovation. Diego can be reached at diego.ponte@unitn.it.

Alessandro Rossi (Ph.D., Organization and Management, University of Udine) is an assistant professor of Business Economics and Management at the Faculties of Economics and Engineering, University of Trento. His current research interests are related to how organizations design and produce complex artefacts, with particular reference to knowledge intensive industries and to the open source/open content paradigm of production. Alessandro can be reached at alessandro. rossi@unitn.it.

Marco Zamarian (Ph.D., Organization and Management, University of Bologna) is an associate professor of Organization Theory and Behavior and Human Resource Management at the Faculty of Economics, University of Trento. His current research interests include organizational learning, knowledge creation and replication in geographically distributed contexts, the impact of IT artefacts on organizational knowledge, industrial clusters, and the evaluation of the effects of public subsidies to the private sector, in particular for technology acquisition and R\&D activities. Marco can be reached at marco.zamarian@unitn.it. 